\title{
Traveling Wave Solutions for some Three-Species Predator-Prey Systems
}

\author{
Jong-Shenq Guo
}

\begin{abstract}
In this paper, we present some recent developments on the application of Schauder's fixed point theorem to the existence of traveling waves for some three-species predator-prey systems. The existence of traveling waves of predator-prey systems is closely related to the invasion phenomenon of some alien species to the habitat of aboriginal species. Three different three-species predator-prey models with different invaded and invading states are presented. In this paper, we focus on the methodology of deriving the convergence of stale tail of wave profiles.
\end{abstract}

\section{Introduction}

In this paper, we are concerned with 3-species predator-prey models for 2-predator-1-prey and 2-prey-1-predator. More precisely, we consider the following systems

$$
\left\{\begin{array}{l}
u_{t}=d_{1} u_{x x}+r_{1} u(-1-u-k v+a w), x \in \mathbb{R}, t>0, \\
v_{t}=d_{2} v_{x x}+r_{2} v(-1-h u-v+a w), x \in \mathbb{R}, t>0, \\
w_{t}=d_{3} w_{x x}+r_{3} w(1-b u-b v-w), x \in \mathbb{R}, t>0,
\end{array}\right.
$$

in which $u, v$ are two predators and $w$ is a single prey; and

$$
\left\{\begin{array}{l}
u_{t}=d_{1} u_{x x}+r_{1} u\left(1-u-k v-b_{1} w\right), x \in \mathbb{R}, t>0, \\
v_{t}=d_{2} v_{x x}+r_{2} v\left(1-h u-v-b_{2} w\right), x \in \mathbb{R}, t>0 \\
w_{t}=d_{3} w_{x x}+r_{3} w(-1+a u+a v-w), x \in \mathbb{R}, t>0
\end{array}\right.
$$

where $u, v$ are two preys and $w$ is a single predator.

The parameters appeared in both systems (1.1) and (1.2) are all positive such that $d_{i}$ denotes the diffusion coefficient, $r_{i}$ is the intrinsic growth rate, and the net growth rate of any predator is

2010 Mathematics Subject Classification. 35K57, 34B40, 92D25, 92D40.

Key words and phrases. predator-prey model, traveling wave, wave speed, upper-lower-solution.

Corresponding author: Jong-Shenq Guo. 
assumed to be negative (which is also normalized to be -1 ) so that any predator cannot survive without the prey(s). Moreover, all preys obey the logistic growth with carrying capacity normalized to be 1 , the conversion rate $a$ is always assumed to satisfy $a>1$ so that the predator(s) can live together with the prey(s), $b\left(b_{i}\right)$ is the predation rate, and $h, k$ are competition coefficients. As for the competition, we consider two weak competing predators in (1.1) so that $0<h, k<1$. For (1.2), there are two cases: either two weak competing preys so that $0<h, k<1$, or a pair of weak-strong competing preys so that $0<h<1<k$.

We are interested in the existence of traveling wave solution in the form

$$
(u, v, w)(x, t):=\left(\phi_{1}, \phi_{2}, \phi_{3}\right)(x+s t)
$$

for some constant $s$ (wave speed) and function $\left(\phi_{1}, \phi_{2}, \phi_{3}\right)$ (wave profiles). Hence, for system (1.1), $\left(s, \phi_{1}, \phi_{2}, \phi_{3}\right)$ satisfies

$$
\left\{\begin{array}{l}
d_{1} \phi_{1}^{\prime \prime}(z)-s \phi_{1}^{\prime}(z)+r_{1} \phi_{1}(z)\left(-1-\phi_{1}-k \phi_{2}+a \phi_{3}\right)(z)=0 \\
d_{2} \phi_{2}^{\prime \prime}(z)-s \phi_{2}^{\prime}(z)+r_{2} \phi_{2}(z)\left(-1-h \phi_{1}-\phi_{2}+a \phi_{3}\right)(z)=0 \\
d_{3} \phi_{3}^{\prime \prime}(z)-s \phi_{3}^{\prime}(z)+r_{3} \phi_{3}(z)\left(1-b \phi_{1}-b \phi_{2}-\phi_{3}\right)(z)=0
\end{array}\right.
$$

for all $z \in \mathbb{R}$. For system (1.2), $\left(s, \phi_{1}, \phi_{2}, \phi_{3}\right)$ satisfies

$$
\left\{\begin{array}{l}
d_{1} \phi_{1}^{\prime \prime}(z)-s \phi_{1}^{\prime}(z)+r_{1} \phi_{1}(z)\left(1-\phi_{1}-k \phi_{2}-b_{1} \phi_{3}\right)(z)=0 \\
d_{2} \phi_{2}^{\prime \prime}(z)-s \phi_{2}^{\prime}(z)+r_{2} \phi_{2}(z)\left(1-h \phi_{1}-\phi_{2}-b_{2} \phi_{3}\right)(z)=0 \\
d_{3} \phi_{3}^{\prime \prime}(z)-s \phi_{3}^{\prime}(z)+r_{3} \phi_{3}(z)\left(-1+a \phi_{1}+a \phi_{2}-\phi_{3}\right)(z)=0
\end{array}\right.
$$

for all $z \in \mathbb{R}$.

Our main goal is to understand the invasion of the alien species into the habitat of the aboriginal species. Therefore, system (1.3) (or, (1.4)) is supplemented with the following asymptotic boundary conditions

$$
\left(\phi_{1}, \phi_{2}, \phi_{3}\right)(-\infty)=\mathcal{O}_{-},\left(\phi_{1}, \phi_{2}, \phi_{3}\right)(\infty)=\mathcal{O}_{+},
$$

where $\mathcal{O}_{ \pm}$are two different constant states of the predator-prey system. We are mainly interested in the so-called monostable waves such that $\mathcal{O}_{-}$is unstable and $\mathcal{O}_{+}$is stable. Hence we call $\mathcal{O}_{-}$ the invaded state and $\mathcal{O}_{+}$the invading state.

Some typical examples of $\mathcal{O}_{-}$:

1. predator-free state:

- $(0,0,1)$ for $(1.1)$, one aboriginal prey and two alien invading predators; 
- $\left(u_{p}, v_{p}, 0\right)$ for (1.2) with $h, k<1$, two aboriginal preys and one alien invading predator, where

$$
u_{p}:=\frac{1-k}{1-h k}, \quad v_{p}:=\frac{1-h}{1-h k} .
$$

2. semi-co-existence state for system (1.2) with $h<1<k$ :

- $E^{*}:=\left(u^{*}, 0, w^{*}\right)$, one weak aboriginal prey and one aboriginal predator with one alien strong intruding prey, where

$$
u^{*}:=\frac{1+b_{1}}{1+a b_{1}}, \quad w^{*}:=\frac{a-1}{1+a b_{1}} ;
$$

- $E_{*}:=\left(0, v_{*}, w_{*}\right)$, one strong aboriginal prey and one aboriginal predator with one alien weak intruding prey, where

$$
v_{*}:=\frac{1+b_{2}}{1+a b_{2}}, \quad w_{*}:=\frac{a-1}{1+a b_{2}} .
$$

Some typical examples of $\mathcal{O}_{+}$are the unique (positive) co-existence state $E_{c}:=\left(u_{c}, v_{c}, w_{c}\right)$ for two weak competing predators of (1.1), two weak competing preys of (1.2), or a pair of weakstrong competing preys of (1.2), and the semi-co-existence states $\left(0, v_{*}, w_{*}\right),\left(u^{*}, 0, w^{*}\right)$ in the case of one aboriginal predator with a pair of weak-strong competing preys for system (1.2). One should distinguish the semi-co-existence states for $\mathcal{O}_{+}$from the ones for $\mathcal{O}_{-}$by their stabilities. The precise conditions on parameters for the stabilities are to be specified later. As for the coexistence state, under certain conditions on the parameters, we have

$$
u_{c}:=\frac{1-k}{1-h k}\left(a w_{c}-1\right), v_{c}:=\frac{1-h}{1-h k}\left(a w_{c}-1\right), w_{c}:=\frac{(1-h k)+b(2-h-k)}{(1-h k)+a b(2-h-k)} .
$$

for system (1.1) when $0<h, k<1$,

$$
\begin{aligned}
& u_{c}:=\frac{(1+b)(1-k)}{(1-h k)+a b(2-h-k)} \\
& v_{c}:=\frac{(1+b)(1-h)}{(1-h k)+a b(2-h-k)} \\
& w_{c}:=\frac{a(2-h-k)-(1-h k)}{(1-h k)+a b(2-h-k)}
\end{aligned}
$$

for system (1.2) when $0<h, k<1$ and $b_{1}=b_{2}=b$, while

$$
u_{c}=\frac{\triangle_{u}}{\triangle}, v_{c}=\frac{\triangle_{v}}{\triangle}, w_{c}=\frac{\triangle_{w}}{\triangle},
$$

where

$$
\triangle_{u}:=-b_{1}(a-1)+b_{2}(a-k)-(k-1), \quad \triangle_{v}:=b_{1}(a-h)-b_{2}(a-1)+(1-h),
$$




$$
\triangle_{w}:=a(2-h-k)-(1-h k), \quad \triangle:=1-h k+a b_{1}(1-h)-a b_{2}(k-1),
$$

for system (1.2) when $0<h<1<k$.

It is well-known that the classical monotone iteration method with the help of super-subsolutions is a powerful method for deriving the existence of wave profiles for monotone systems. But, it cannot be applied to predator-prey systems, due to the fact that predator-prey systems are not monotone system (no comparison principle). However, an application of Schauder's fixed point theorem with the help of so-called generalized upper-lower-solutions has been proved to be very successful in the derivation of wave profiles for non-monotone systems. For this aspect, we refer the reader to $[21,22,11,14,15,17,12,16,18,4,24]$ for 2 -species case and to $[6,13,19,23,20$, 1] for 3-species case on predator-prey systems. Of course, these two methods work well for either cases are under the condition that a suitable pair of super-sub (or upper-lower) solutions can be found. This is actually one of the difficulties in applying these two methods and it is non-trivial by no means.

Another difficulty in applying the latter method to non-monotone systems is the derivation of the convergence of stable tail of wave profiles. In this aspect, there are at least two classical methods, namely, the method of contracting rectangles and an application of Lyapunov functions argument. This issue shall be the main focus of this paper.

The rest of this paper is organized as follows. In section 2 , we present the main results obtained recently in $[10,2,3]$ for predator-prey systems (1.1) and (1.2), respectively. This includes the existence and non-existence of traveling waves. It is worth to mention that all of these results give the linear determinacy of minimal wave speeds. Then, in section 3 , we briefly discuss the application of Schauder's fixed point theorem along with the definition of generalized upperlower-solutions to the derivation of the existence of wave profiles. Finally, the discussion on the derivation of the convergence of stable wave tail is given in section 4 . For the derivation of nonexistence results, we omit is here and refer the reader to $[10,2,3]$.

\section{Main results}

We give some main results obtained in $[10,2,3]$ in this section. Set $\Phi:=\left(\phi_{1}, \phi_{2}, \phi_{3}\right)$.

First, for system (1.1) with a single aboriginal prey, we have

Theorem 2.1 ([10]). Let $\tilde{s}:=\max \left\{2 \sqrt{d_{1} r_{1}(a-1)}, 2 \sqrt{d_{2} r_{2}(a-1)}\right\}$ and assume

$$
a>1, \quad 0<h, k<1, \quad 0<b<\frac{1}{2(a-1)} .
$$

Then there is a bounded positive solution $\Phi$ of $(1.3)$ such that $\Phi(-\infty)=(0,0,1)$, if $s \geq \tilde{s}$. Moreover, $\Phi(\infty)=\left(u_{c}, v_{c}, w_{c}\right)$, if we further assume that

$$
0<b<\frac{1}{2 a} \min \{1-h, 1-k\} .
$$


On the other hand, there is no positive solution of (1.3) with boundary condition (1.5), if $s<\tilde{s}$.

Secondly, for system (1.2) with two weak competing aboriginal preys, we have

Theorem 2.2 ([2]). Given $h, k \in(0,1)$. Let $\hat{s}:=2 \sqrt{d_{3} r_{3}\left[a\left(u_{p}+v_{p}\right)-1\right]}$ and consider

$$
\mathcal{O}_{-}=\left(u_{p}, v_{p}, 0\right), \quad \mathcal{O}_{+}=\left(u_{c}, v_{c}, w_{c}\right)
$$

Assume

$$
\begin{aligned}
& a>\frac{2}{2-h-k}, b_{1}=b_{2}=b, \\
& 0<b<\min \left\{\frac{1-k}{2 a-1}, \frac{1-h}{2 a-1}, \frac{a(2-h-k)-2}{2 a(2 a-1)}\right\} .
\end{aligned}
$$

Then, for $s \geq \hat{s}$, under the condition

$$
\max \left\{\frac{d_{1}}{2}, \frac{d_{2}}{2}\right\} \leq d_{3} \leq \min \left\{d_{1}, d_{2}\right\},
$$

system (1.4) has a solution $\Phi$ such that (1.5) holds. Moreover, there is no positive solution for (1.4)(1.5), if $s<\hat{s}$.

The case for a pair of weak-strong competing preys is more complicated. We shall only consider the equal-diffusion case so that $0<h<1<k$ and $d_{1}=d_{2}=d_{3}$. Set

$$
\beta^{*}=1-h u^{*}-b_{2} w^{*}, \beta_{*}=1-k v_{*}-b_{1} w_{*},
$$

or equivalently

$$
\begin{aligned}
& \beta^{*}=\frac{b_{1}(a-h)-b_{2}(a-1)+(1-h)}{1+a b_{1}}, \\
& \beta_{*}=\frac{-b_{1}(a-1)+b_{2}(a-k)-(k-1)}{1+a b_{2}} .
\end{aligned}
$$

Also, set $s^{*}:=2 \sqrt{d_{2} r_{2} \beta^{*}}$, if $\beta^{*}>0$; and $s_{*}:=2 \sqrt{d_{1} r_{1} \beta_{*}}$, if $\beta_{*}>0$.

For the weak competing prey as an aboriginal species, we have more complete picture as follows.

Theorem 2.3 ([3]). Suppose that $0<h<1<k, d_{1}=d_{2}=d_{3}$, and $\beta^{*}>0$. Assume further that

$$
r_{2} \beta^{*} \geq \max \left\{r_{3}, r_{1}\left[k+b_{1}(2 a-1)\right]\right\} .
$$


Then system (1.4) has a bounded positive solution $\Phi$ satisfying $\Phi(-\infty)=\left(u^{*}, 0, w^{*}\right)$ for any $s \geq$ $s^{*}$. Moreover, $\left(\phi_{1}, \phi_{2}, \phi_{3}\right)(\infty)=\left(0, v_{*}, w_{*}\right)$, if $\beta_{*}<0$ and

$$
a>\frac{1}{1-h}, \quad b_{2}<\frac{a(1-h)-1}{a(2 a-1)}
$$

while $\Phi(\infty)=\left(u_{c}, v_{c}, w_{c}\right)$, if the positive co-existence state exists and

$$
k \sqrt{\frac{b_{2}}{b_{1}}}+h \sqrt{\frac{b_{1}}{b_{2}}}<2 .
$$

For the case of aboriginal strong competing prey, we have

Theorem 2.4 ([3]). Suppose that $0<h<1<k, d_{1}=d_{2}=d_{3}$, and $\beta_{*}>0$. Assume further that

$$
r_{1} \beta_{*} \geq \max \left\{r_{3}, r_{2}\left[h+b_{2}(2 a-1)\right]\right\}
$$

Then system (1.4) has a bounded positive solution $\Phi$ satisfying $\Phi(-\infty)=\left(0, v_{*}, w_{*}\right)$ for any $s \geq s_{*}$. Moreover, we have

$$
\liminf _{z \rightarrow+\infty} \phi_{1}(z)>0, \liminf _{z \rightarrow+\infty} \phi_{3}(z)>0
$$

Furthermore, if the positive co-existence state $\left(u_{c}, v_{c}, w_{c}\right)$ exists and (2.4) is enforced, then $\Phi(\infty)=$ $\left(u_{c}, v_{c}, w_{c}\right)$.

It is left open for the existence of traveling waves with $\mathcal{O}_{-}=\left(0, v_{*}, w_{*}\right)$ and $\mathcal{O}_{+}=\left(u^{*}, 0, w^{*}\right)$. In other words, we are not sure whether the weak competing intruding prey can replace the aboriginal strong competing prey in system (1.2).

That either prey invading the environment at least at the corresponding speed $s^{*}$ or $s_{*}$ is shown by the following non-existence of waves.

Theorem 2.5 ([3]). The following statements hold:

1. Assume that $\beta^{*}>0$, hence $s^{*}>0$. Then no positive solutions of (1.4) and (1.5) with $\mathcal{O}_{-}=\left(u^{*}, 0, w^{*}\right)$ and $\mathcal{O}_{+} \in\left\{\left(u_{c}, v_{c}, w_{c}\right),\left(0, v_{*}, w_{*}\right)\right\}$ exist for $s<s^{*}$.

2. Assume that $\beta_{*}>0$, hence $s_{*}>0$. Then no positive solutions of (1.4) and (1.5) with $\mathcal{O}_{-}=\left(0, v_{*}, w_{*}\right)$ and $\mathcal{O}_{+} \in\left\{\left(u_{c}, v_{c}, w_{c}\right),\left(u^{*}, 0, w^{*}\right)\right\}$ exist for $s<s_{*}$. 


\section{Existence of wave profiles}

In this section, we briefly discuss method of deriving the existence of wave profiles by the application of Schauder's fixed point theorem.

First, we introduce the notion of generalized upper-lower solutions. Since it is similar for system (1.4), we only give the definition for system (1.3) as follows.

Definition 1. Given $s>0$. Continuous functions $\left(\bar{\phi}_{1}, \bar{\phi}_{2}, \bar{\phi}_{3}\right)$ and $\left(\underline{\phi}_{1}, \underline{\phi}_{2}, \underline{\phi}_{3}\right)$ defined on $\mathbb{R}$ are called a pair of generalized upper-lower solutions of (1.3) if $\bar{\phi}_{i}^{\prime \prime}, \underline{\phi}_{i}^{\prime \prime}, \bar{\phi}_{i}^{\prime}, \underline{\phi}_{i}^{\prime}, i=1,2,3$, are bounded functions such that the following inequalities hold:

$$
\begin{aligned}
& \mathcal{U}_{1}(z):=d_{1} \bar{\phi}_{1}^{\prime \prime}(z)-s \bar{\phi}_{1}^{\prime}(z)+r_{1} \bar{\phi}_{1}(z)\left[-1-\bar{\phi}_{1}(z)-k \underline{\phi}_{2}(z)+a \bar{\phi}_{3}(z)\right] \leq 0, \\
& \mathcal{U}_{2}(z):=d_{2} \bar{\phi}_{2}^{\prime \prime}(z)-s \bar{\phi}_{2}^{\prime}(z)+r_{2} \bar{\phi}_{2}(z)\left[-1-h \underline{\phi}_{1}(z)-\bar{\phi}_{2}(z)+a \bar{\phi}_{3}(z)\right] \leq 0, \\
& \mathcal{U}_{3}(z):=d_{3} \bar{\phi}_{3}^{\prime \prime}(z)-s \bar{\phi}_{3}^{\prime}(z)+r_{3} \bar{\phi}_{3}(z)\left[1-b \underline{\phi}_{1}(z)-b \underline{\phi}_{2}(z)-\bar{\phi}_{3}(z)\right] \leq 0, \\
& \mathcal{L}_{1}(z):=d_{1} \underline{\phi}_{1}^{\prime \prime}(z)-s \underline{\phi}_{1}^{\prime}(z)+r_{1} \underline{\phi}_{1}(z)\left[-1-\underline{\phi}_{1}(z)-k \bar{\phi}_{2}(z)+a \underline{\phi}_{3}(z)\right] \geq 0, \\
& \mathcal{L}_{2}(z):=d_{2} \underline{\phi}_{2}^{\prime \prime}(z)-s \underline{\phi}_{2}^{\prime}(z)+r_{2} \underline{\phi}_{2}(z)\left[-1-h \bar{\phi}_{1}(z)-\underline{\phi}_{2}(z)+a \underline{\phi}_{3}(z)\right] \geq 0, \\
& \mathcal{L}_{3}(z):=d_{3} \underline{\phi}_{3}^{\prime \prime}(z)-s \underline{\phi}_{3}^{\prime}(z)+r_{3} \underline{\phi}_{3}(z)\left[1-b \bar{\phi}_{1}(z)-b \bar{\phi}_{2}(z)-\underline{\phi}_{3}(z)\right] \geq 0
\end{aligned}
$$

for $z \in \mathbb{R} \backslash E$ with some finite set $E=\left\{z_{1}, z_{2}, \ldots, z_{m}\right\}$.

The following proposition gives the existence of solution $\left(\phi_{1}, \phi_{2}, \phi_{3}\right)$ to system (1.3).

Proposition 3.1. Given $s>0$. Suppose that system (1.3) has a pair of generalized upper-lower solutions $\left(\bar{\phi}_{1}, \bar{\phi}_{2}, \bar{\phi}_{3}\right)$ and $\left(\underline{\phi}_{1}, \underline{\phi}_{2}, \underline{\phi}_{3}\right)$ such that

$$
\begin{aligned}
& \underline{\phi}_{i}(z) \leq \bar{\phi}_{i}(z), \forall z \in \mathbb{R}, i=1,2,3, \\
& \bar{\phi}_{i}^{\prime}\left(z_{j}^{+}\right) \leq \bar{\phi}_{i}^{\prime}\left(z_{j}^{-}\right), \underline{\phi}_{i}^{\prime}\left(z_{j}^{-}\right) \leq \underline{\phi}_{i}^{\prime}\left(z_{j}^{+}\right), \forall z_{j} \in E, i=1,2,3 .
\end{aligned}
$$

Then system (1.3) has a solution $\left(\phi_{1}, \phi_{2}, \phi_{3}\right)$ such that $\underline{\phi}_{i} \leq \phi_{i} \leq \bar{\phi}_{i}, i=1,2,3$.

The proof of Proposition 3.1 is by now standard, we only give an outline as follows. The proof can be divided into the following steps:

1. Transform the differential system to an appropriate integral system with integral operator $P$ so that the existence of a solution to the differential system is reduced to a fixed point of the integral operator $P$.

2. Since we have a pair of upper-lower solutions, we can define the set

$$
\Sigma:=\left\{\left(\phi_{1}, \phi_{2}, \phi_{3}\right) \mid \underline{\phi}_{i} \leq \phi_{i} \leq \bar{\phi}_{i}, i=1,2,3\right\}
$$

and verify that $P$ maps $\Sigma$ into itself, using the definition of upper-lower solutions, (3.2) and the partial monotone property of $P$. 
3. Show the operator $P$ is completely continuous with respect to a suitable complete weighted normed space on $\Sigma$.

4. Finally, Schauder's fixed point theorem gives a fixed point of $P$ in $\Sigma$ which is the desired solution of the original differential system.

With Proposition 3.1, the next task is to construct a pair of suitable generalized upper-lower solutions for each case. We refer the reader to $[10,2,3]$ for the detailed constructions and their verifications for various cases in our main theorems. A few remarks are made as follows.

First, a suitable pair of generalized upper-lower solutions are not always available, just like the construction of Lyapunov functions in the study of asymptotic behavior of solutions to evolution equations. Hence the construction of generalized upper-lower solutions is by no means trivial in general. Certain restrictions on the parameters are required, but we believe that these restrictions might be just technical. In fact, as one can expect, the more number of aboriginal species the more difficult to construct generalized upper-lower solutions. Nevertheless usually one can construct generalized upper-lower solutions to capture the asymptotic behavior at the unstable tail $\mathrm{O}^{-}$. So, with a suitable generalized upper-lower solutions, it remains to derive the convergence of wave profiles to the stable state at the stable tail.

\section{Derivation of the stable tail limit}

In deriving the stable tail limit, there are at least two classical methods, namely, the method of contracting rectangles and the method of Lyapunov functions argument. In the sequel, we let

$$
\phi_{i}^{-}:=\liminf _{z \rightarrow \infty} \phi_{i}(z), \phi_{i}^{+}:=\limsup _{z \rightarrow \infty} \phi_{i}(z), i=1,2,3,
$$

for a derived wave profile $\left(\phi_{1}, \phi_{2}, \phi_{3}\right)$.

To illustrate the method of contracting rectangles (MCR for short), we first consider the case for

$$
\left(\phi_{1}, \phi_{2}, \phi_{3}\right)(\infty)=\left(u_{c}, v_{c}, w_{c}\right) .
$$

The first ingredient of MCR is to construct a sequence of shrinking rectangles $\{Q(\theta)\}_{\theta \in[0,1]}$ in $(0, \infty)^{3}$ such that $Q(\theta) \downarrow\left(u_{c}, v_{c}, w_{c}\right)$ as $\theta \uparrow 1^{-}$. Set

$$
Q(\theta):=\left[m_{1}(\theta), M_{1}(\theta)\right] \times\left[m_{2}(\theta), M_{2}(\theta)\right] \times\left[m_{3}(\theta), M_{3}(\theta)\right], \theta \in[0,1] .
$$

Then one needs the properties

$$
m_{i}(\theta) \uparrow m_{i}(1) \text { and } M_{i}(\theta) \downarrow M_{i}(1)=m_{i}(1) \text { as } \theta \uparrow 1^{-}, i=1,2,3 \text {. }
$$


The second ingredient of MCR is to verify $A \neq \emptyset$ (usually, $0 \in A$ ) and $\sup A=1$, where

$$
A:=\left\{\theta \in[0,1) \mid m_{i}(\theta)<\phi_{i}^{-} \leq \phi_{i}^{+}<M_{i}(\theta), i=1,2,3\right\} .
$$

In fact, it is not too hard to find a sequence of contracting rectangles. The main difficulty in applying MCR is to verify that $\sup A=1$.

Before applying the method of contracting rectangles, we derive $\phi_{i}^{-} \geq b_{i}$ for some positive constant $b_{i}, i=1,2$, using $\phi_{3}^{-}>0$. See [10, Lemma 4.1].

To prove (4.1) in Theorem 2.1, a new form of contracting rectangles is introduced as follows. For $\theta \in[0,1]$, we define

$$
\begin{aligned}
& m_{1}(\theta):=\theta u_{c}+(1-\theta)\left(b_{1}-\varepsilon\right), M_{1}(\theta):=(1-\theta)(a-1+\varepsilon)+\theta u_{c}, \\
& m_{2}(\theta):=\theta v_{c}+(1-\theta)\left(b_{2}-\varepsilon\right), M_{2}(\theta):=(1-\theta)(a-1+\varepsilon)+\theta v_{c}, \\
& m_{3}(\theta):=\theta w_{c}+(1-\theta)\left(1-a \beta-\varepsilon^{2}\right), M_{3}(\theta):=(1-\theta)\left(1+\varepsilon^{2}\right)+\theta w_{c},
\end{aligned}
$$

where $\varepsilon$ is a small positive constant (cf. [10, (4.3)]). Notice that in the expressions of $m_{3}(\theta)$ and $M_{3}(\theta)$ there appear the term $\varepsilon^{2}$ instead of $\varepsilon$. Then $\sup A=1$ can be derived by a contradiction argument.

To derive $\sup A=1$, it requires $m_{i}(\theta)>0, i=1,2,3$, and

$$
\begin{aligned}
& -1-m_{1}(\theta)-k M_{2}(\theta)+a m_{3}(\theta)>0, \quad-1-M_{1}(\theta)-k m_{2}(\theta)+a M_{3}(\theta)<0, \\
& -1-h M_{1}(\theta)-m_{2}(\theta)+a m_{3}(\theta)>0, \quad-1-h m_{1}(\theta)-M_{2}(\theta)+a M_{3}(\theta)<0 \\
& 1-b M_{1}(\theta)-b M_{2}(\theta)-m_{3}(\theta)>0, \quad 1-b m_{1}(\theta)-b m_{2}(\theta)-M_{3}(\theta)<0,
\end{aligned}
$$

for all $\theta \in(0,1)$. Hence (4.1) is proved. We remark that the positivity (or negativity) in (4.2)-(4.4) is the key to the success of MCR.

A similar new form of contracting rectangles are used to prove (4.1) in Theorem 2.2. We refer the reader to [2] for the details.

To derive $\left(\phi_{1}, \phi_{2}, \phi_{3}\right)(\infty)=\left(0, v_{*}, w_{*}\right)$ in Theorem 2.3, the full 3-d MCR is not applicable, due to that $\phi_{1}^{-}>0$ cannot be expected. In [3], we introduce an idea of dimension reduction and consider a sequence of 2 - $\mathrm{d}$ rectangles for $\left(\phi_{2}, \phi_{3}\right)$ to verify $\left(\phi_{2}, \phi_{3}\right)(\infty)=\left(v_{*}, w_{*}\right)$ first. Then $\phi_{1}(\infty)=0$ is reduced.

Since the constructed lower solutions vanish at the right-hand side of the horizontal axis, we need to derive $\phi_{i}^{-}>0, i=2,3$, before applying the method of 2 - $\mathrm{d}$ contracting rectangles. This is done by applying some dynamical system arguments from persistence theory (cf. [8, 7]). Actually, this very technical part is one of the main contributions of the work [3]. We refer the reader to [3] for the details. Once we have $\phi_{2}^{-}>0$ and $\phi_{3}^{-}>0$, then the above procedures of (3-d) MCR can be applied with some modifications to derive $\left(\phi_{2}, \phi_{3}\right)(\infty)=\left(v_{*}, w_{*}\right)$. In the 
derivation of (4.3)-(4.4) (for the 2-d MCR), the corresponding quantity to $m_{1}(\theta)$ is set to be zero and the corresponding quantity to $M_{1}(\theta)$ (i.e., the upper bounds of $\phi_{1}^{+}$) can be estimated such that $M_{1}(\theta) \downarrow 0$ as $\theta \uparrow 1^{-}$. Therefore, $\phi_{1}(\infty)=0$ follows immediately.

To derive $\left(\phi_{1}, \phi_{2}, \phi_{3}\right)(\infty)=\left(u_{c}, v_{c}, w_{c}\right)$ in Theorem 2.3, recall from the above that $\phi_{2}^{-}>0$ and $\phi_{3}^{-}>0$. Moreover, we can derive $\phi_{1}^{-}>0$, by a contradiction argument using the following proposition.

Proposition $4.1([5,9,3])$. Any entire in time solution $(v, w)$ of system

$$
\left\{\begin{array}{l}
v_{t}=d_{2} v_{x x}+r_{2} v\left(1-v-b_{2} w\right), \\
w_{t}=d_{3} w_{x x}+r_{3} w(-1+a v-w),
\end{array}\right.
$$

such that

$$
0<\min \left\{\inf _{\mathbb{R}^{2}} v, \inf _{\mathbb{R}^{2}} w\right\}<\max \left\{\sup _{\mathbb{R}^{2}} v, \sup _{\mathbb{R}^{2}} w\right\}<+\infty,
$$

must satisfy that $v \equiv v_{*}$ and $w \equiv w_{*}$.

Unfortunately, the method of contracting rectangles does not work (neither 3-d nor 2-d), even we have $\phi_{i}^{-}>0$ for all $i$. Therefore, we apply a Lyapunov argument using

Proposition $4.2([3])$. Assume that $E_{c}=\left(u_{c}, v_{c}, w_{c}\right)$ exists and

$$
k \sqrt{\frac{b_{2}}{b_{1}}}+h \sqrt{\frac{b_{1}}{b_{2}}}<2 .
$$

Let $(u, v, w)=(u, v, w)(x, t)$ be a bounded entire in time solution of (1.2) such that

$$
\min \left\{\inf _{\mathbb{R}^{2}} u, \inf _{\mathbb{R}^{2}} v, \inf _{\mathbb{R}^{2}} w\right\}>0
$$

Then $(u, v, w) \equiv\left(u_{c}, v_{c}, w_{c}\right)$.

For a proof of Proposition 4.2, see [3, Lemma 4.7].

Finally, the proof of $\left(\phi_{1}, \phi_{2}, \phi_{3}\right)(\infty)=\left(u_{c}, v_{c}, w_{c}\right)$ in Theorem 2.4 is similar to that in Theorem 2.3. Unfortunately, we were unable to derive $\left(\phi_{1}, \phi_{2}, \phi_{3}\right)(\infty)=\left(u^{*}, 0, w^{*}\right)$ as in Theorem 2.3, if $\beta^{*}<0$ and certain restrictions on the parameters are imposed. If we can prove $\left(\phi_{1}, \phi_{2}, \phi_{3}\right)(\infty)=\left(u^{*}, 0, w^{*}\right)$, then it corresponds to that the weak intruding prey can replace the aboriginal strong prey to live with the aboriginal predator. This is a very interesting question and is left open.

\section{Acknowledgments}

This work was partially supported by the Ministry of Science and Technology of Taiwan under the grant 108-2115-M-032-006-MY3. 


\section{References}

[1] Z. Bi and S. Pan, Dynamics of a predator-prey system with three species, Bound. Value. Probl. (2018) 2018:162.

[2] Y.-S. Chen and J.-S. Guo, Traveling wave solutions for a three-species predatorprey model with two aborigine preys, Japan J. Industrial and Applied Mathematics, https://doi.org/10.1007/s13160-020-00445-9.

[3] Y.-S. Chen, T. Giletti and J.-S. Guo, Persistence of preys in a diffusive three species predatorprey system with a pair of strong-weak competing preys, arXiv:2008.11939.

[4] Y.-Y. Chen, J.-S. Guo and C.-H. Yao, Traveling wave solutions for a continuous and discrete diffusive predator-prey model, J. Math. Anal. Appl., 445 (2017), 212-239.

[5] Y. Du and S.-B. Hsu, A diffusive predator-prey model in heterogeneous environment, J. Differential Equations, 203 (2004), 331-364.

[6] Y. Du and R. Xu, Traveling wave solutions in a three-species food-chain model with diffusion and delays, Int. J. Biomath., 5 (2012), 1250002, 17 pp.

[7] A. Ducrot, T. Giletti, J.-S. Guo and M. Shimojo, Asymptotic spreading speeds for a predatorprey system with two predators and one prey, Nonlinearity, https://doi.org/10.1088/1361$6544 / \mathrm{abd} 289$.

[8] A. Ducrot, T. Giletti and H. Matano, Spreading speeds for multidimensional reactiondiffusion systems of the prey-predator type, Calc. Var. Partial Differential Equations, 58 (2019), no. 4, Paper No. 137, 34 pp.

[9] A. Ducrot and J.-S. Guo, Asymptotic behavior of solutions to a class of diffusive predatorprey systems, J. Evolution Equations, 18 (2018), 755-775.

[10] J.-S. Guo, K.-I. Nakamura, T. Ogiwara and C.-C. Wu, Traveling wave solutions for a predatorprey system with two predators and one prey, Nonlinear Analysis: Real World Applications, 54 (2020), 103111, 13 pp.

[11] J. Huang, G. Lu and S. Ruan, Existence of traveling wave solutions in a diffusive predatorprey model, J. Math. Biol., 46 (2003), 132-152.

[12] W. Huang, Traveling wave solutions for a class of predator-prey systems, J. Dynam. Differential Equations, 24 (2012), 633-644. 
[13] Y.L. Huang and G. Lin, Traveling wave solutions in a diffusive system with two preys and one predator, J. Math. Anal. Appl., 418 (2014), 163-184.

[14] J. Huang and X. Zou, Existence of traveling wave fronts of delayed reaction-diffusion systems without monotonicity, Disc. Cont. Dyn. Systems, 9 (2003), 925-936.

[15] W.T. Li, G. Lin and S. Ruan, Existence of traveling wave solutions in delayed reactiondiffusion systems with applications to diffusion-competition systems, Nonlinearity, 19 (2006), 1253-1273.

[16] G. Lin, Invasion traveling wave solutions of a predator-prey system, Nonlinear Anal., 96 (2014), 47-58.

[17] G. Lin, W.T. Li and M. Ma, Traveling wave solutions in delayed reaction diffusion systems with applications to multi-species models, Disc. Cont. Dyn. Systems - B, 13 (2010), 393-414.

[18] G. Lin and S. Ruan, Traveling wave solutions for delayed reaction-diffusion systems and applications to diffusive Lotka-Volterra competition models with distributed delays, J. Dyn. Diff. Equat., 26 (2014), 583-605.

[19] J.-J. Lin, W. Wang, C. Zhao and T.-H. Yang, Global dynamics and traveling wave solutions of two predators-one prey models, Discrete and Continuous Dynamical System - B, 20 (2015), 1135-1154.

[20] J.-J. Lin and T.-H. Yang, Traveling wave solutions for a diffusive three-species intraguild predation model, Int. J. Biomath., 11 (2018), 1850022, 27 pp.

[21] S. Ma, Traveling wavefronts for delayed reaction-diffusion systems via a fixed point theorem, J. Differential Equations, 171 (2001), 294-314.

[22] J. Wu and X. Zou, Traveling wave fronts of reaction-diffusion systems with delay, J. Dynam. Differential Equations, 13 (2001), 651-687.

[23] T. Zhang, Minimal wave speed for a class of non-cooperative reaction-diffusion systems of three equations, J. Differential Equations, 262 (2017), 4724-4770.

[24] T. Zhang and Y. Jin, Traveling waves for a reaction-diffusion-advection predator-prey model, Nonlinear Analysis: Real World Applications, 36 (2017), 203-232.

Jong-Shenq Guo Department of Mathematics, Tamkang University, Tamsui, New Taipei City 251301, Taiwan

E-mail: jsguo@mail.tku.edu.tw 